\title{
Whole Genome Sequencing: An Efficient Approach in Food Safety Management System
}

Hedayat Hossieni*

1- National Nutrition \& Food Technology Research Institute, Faculty of Nutrition Sciences and Food Technology, Shahid Beheshti University of Medical Sciences, Tehran, Iran

$\mathrm{F}$ ood safety is still a worldwide concern so that, in many countries, production and distribution of safe and nutritious food is one of the most important problems; meanwhile, the right to have healthy food is obvious.

Recently food control systems have improved in many countries. However, while progress has been made, in 2010, the estimated global burden is reportedly about 600 million foodborne illnesses and 420,000 deaths (1). Application of new technologies for empowering detection and prevention of food born diseases is important to decline the burden of food-borne diseases (2); hence, empowerment of food safety system through developing the laboratory technologies from non-automated DNA sequencing methods to more rapid, automated sequencing methods such as whole genome sequencing (WGS) with the ability to sequence the entire genomes would have great impact on food safety management system.

WGS makes precise, rapid and helpful information to prevent food-borne diseases. In spite of the certain limitations in the field of technical and facilities infrastructures for implementation of this technology, performance and traceability of food safety management systems at national and international levels can be improved through application of whole genome sequencing in food, water, environmental and clinical samples continuously.

WGS is a new technology that can identify the complete DNA sequence of a food-borne pathogen genome. This laboratory methodology has an important role in improvement of food safety through making source tracking and traceability, source attribution, food system monitoring, disease surveillance, outbreak investigation, identification of transmission pathways and monitoring food-borne diseases (3).

In addition, WGS is helpful in reducing food waste due to contamination, and thereby has a serious effect on food security. While WGS can properly contribute to improving food safety and security issues, lack of experienced experts for technical and bioinformatics purposes, providing necessary infrastructures for storage and data sharing facilities, and interpretation of laboratory data in epidemiological evidence are some barriers in globalization of this new method, and so its application remains relatively limited, especially in the developing and transitional countries (4). Due to the importance of technical aspects and infrastructures in implementation of WGS, decisions about when and where to use this technology for strengthening national food control systems need comprehensive assessment by each country $(5,6)$.

WGS has the potential to improve the performance of food safety management systems through food-borne diseases' surveillance, food tracing and monitoring, and detecting the source of contamination; in the meantime, there are some challenges in application of WGS to become a globally accepted methodology for identification of food-borne pathogens.

\section{Financial disclosure}

The authors declare no financial interest.

\section{References}

1. WHO. 2015. WHO estimates of the global burden of foodborne diseases: foodborne disease burden epidemiology reference group 2007-2015. Geneva, Switzerland. Publication (available at http://apps. who.int/iris/bitstream/10665/199350/1/9789241565165_ eng.pdf). Accessed 17 March 2016.

2. Hosseini H, Jamshidi A, Bassami M, Khaksar R, Zeynali T, Mousavi-Khaneghah A. et al. Isolation,

*Address for correspondence: Hedayat Hossieni, Prof, National Nutrition \& Food Technology Research Institute, Faculty of Nutrition Sciences and 
identification and virulence gene profiling of Escherichia coli O157:H7 in retail doner kebabs, Iran. J Food Safety, 2013; 33(4):489-96.

3. Lambert D, Carrillo CD, Koziol AG, Manninger P, Blais B.W. GeneSippr: A rapid whole-genome approach for the identification and characterization of foodborne pathogens such as priority Shiga Toxigenic Escherichia coli. PlosOne. 2015; 10(4): 122-28.

4. Zhang S, Yin Y, Jones MB, Zhang Z, Kaiser BLD, Dinsmore BA. Salmonella serotype determination utilizing high-throughput genome sequencing data. J Clinical Microbiol 2015; 53(5): 1685-92.

5. Pielaat A, Boer MP, Wijnands LM, van Hoek AH, Bouw E, Barker GC. First step in using molecular data for microbial food safety risk assessment; hazard identification of Escherichia coli O157:H7 by coupling genomic data with in vitro adherence to human epithelial cells. Int J Food Microbiol 2015; 213: 13038 .

6. ECDC (European Centre for Disease Prevention and Control). 2015. Expert opinion on the introduction of next-generation typing methods for food- and waterborne diseases in the EU and EEA. Stockholm, Sweden. Doi: 10.2900/4536411. Publication (available at http://ecdc.europa.eu/en/publications/Publications/ food-and-waterborne-diseases-next-generation- typingmethods.pdf). Accessed 17 March 2016. 